\title{
A Microchannel Neural Interface ASIC
}

\author{
Henry Lancashire ${ }^{1}$, Dai Jiang ${ }^{2}$, Andreas Demosthenous ${ }^{2}$ and Nick Donaldson ${ }^{3}$ \\ ${ }^{1}$ Department of Materials and Tissue, ${ }^{2}$ Department of Electronic and Electrical Engineering, \\ ${ }^{3}$ Department of Medical Physics and Bioengineering, University College London, London, WC1E 6BT, United Kingdom. \\ \{henry.lancashire.10; d.jiang; a.demosthenous; n.donaldson\}@ucl.ac.uk
}

\begin{abstract}
This paper presents an application specific integrated circuit (ASIC) for use in a three-dimensional microchannel neural interface. The device is assembled with seven stacked ASICs with silicone microchannels instrumented in between. Each ASIC comprises tripolar electrodes for seven channels allowing recording or stimulation from any one of the 49 microchannels. The ASIC is implemented in $0.35-\mu \mathrm{m}$ high-voltage CMOS technology and occupies an active area of $4 \mathrm{~mm}^{2}$. The device has been tested demonstrating recording of $1 \mathrm{mV}$ signals, and current controlled stimulation in the range of $5 \mu \mathrm{A}$ to $500 \mu \mathrm{A}$ (with $40 \mathrm{~V}$ compliance) and up to $50 \mathrm{kHz}$ stimulation frequency. The device overcomes limits on numbers of connected microchannels in previous designs.
\end{abstract}

Keywords-Bioelectronics, electrodes, microchannels, neural interfaces.

\section{INTRODUCTION}

Upper limb amputees report dissatisfaction with current commercial prostheses [1]. Complaints include limited degrees of freedom and unintuitive control [2]. Interfacing with residual peripheral nerves may make intuitive control of amputation prostheses possible [3]. Many devices for recording from and stimulating the peripheral nervous system have been investigated [4], and one key approach to re-establishing intuitive sensory and motor function is using highly selective neural interfaces [5]. The challenges for such neural interfaces include the interference between channels during multi-channel stimulation, the small magnitude of the extracellular action potential in fragile, dynamic environment, and the signal stability often degraded by scar tissue and fibrosis formed around the electrodes.

Microchannel neural interfaces (MNIs) are one solution to providing a highly selective interface with the nervous system. MNI design helps overcome some of the challenges of nerve interfaces, including low signal amplitude and cross-talk [6]. MNIs comprise an array of small diameter microchannels (typically $\varnothing<200 \mu \mathrm{m}$ and up to $5 \mathrm{~mm}$ in length) into which peripheral nerve axons are placed or encouraged to regenerate. The electrically insulating microchannel acts to: increase signal amplitude by restricting the extracellular space [7]; reduce crosstalk by physically separating axons; increase the length of axon from which signals can be recorded, making recordings independent of node of Ranvier position; amplify the magnitude of the action potential by the narrow channel; and reduce the required stimulus currents. Evidence from in-vivo results also suggests minimal fibrosis and inflammation [7]-[9]. Selectivity in MNI devices partly depends upon the number of

This work was funded by the Engineering and Physical Sciences Research Council (UK) under their Doctoral Prize Fellowship scheme.

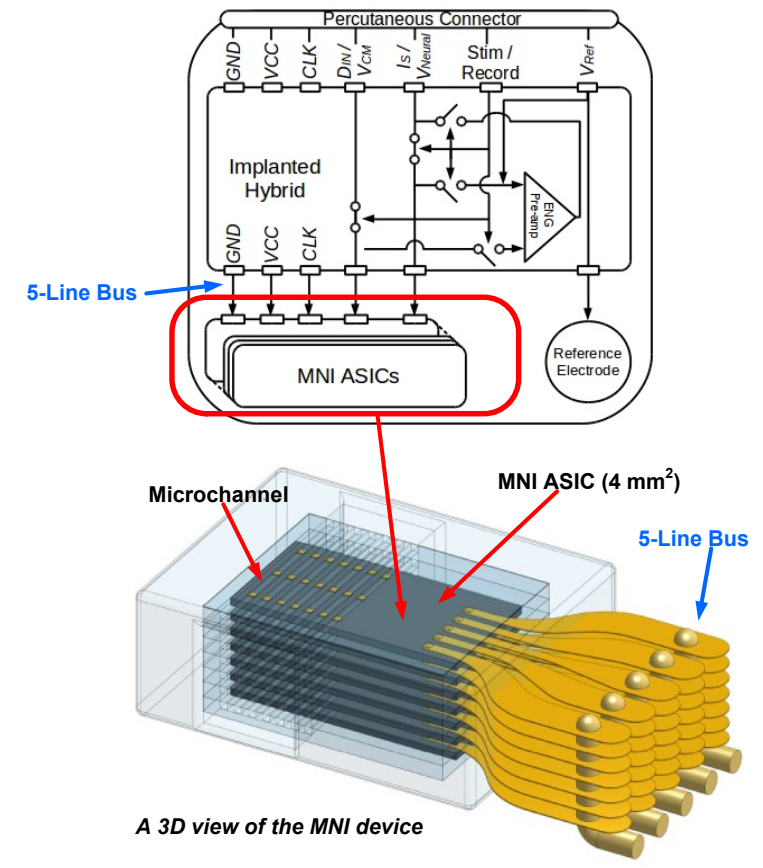

Figure 1. Overall architecture of the MNI implant.

microchannels. To achieve high numbers of channels, MNIs have been manufactured in three-dimensions (3D) by several methods [6], [8], [9].

To date MNIs have used passive conductors within the microchannel array, which sets a limit on the number of channels, since each channel will require at least one connecting wire. We propose to use ASICs in place of passive conductors to significantly reduce the number of connections required by multiplexing electrode sites. This paper describes the MNI ASIC developed comprising on-chip electrodes, formed from the standard bonding pads, for seven microchannel arrays and the associated multiplexing circuits. Multiple ASICs are used to construct a MNI device with 49 microchannels providing interface to 49 possible axon bundles. This is a significant increase in the number of channels despite the fact that the number of connecting wires is reduced to a 5-line bus. Circuit design and device fabrication procedures are described.

\section{SYSTEM AND ASIC ARCHITECTURE}

\section{A. Overall System}

The system architecture is shown in Fig. 1 alongside a 3D view of the final MNI device, where seven MNI ASICs are 


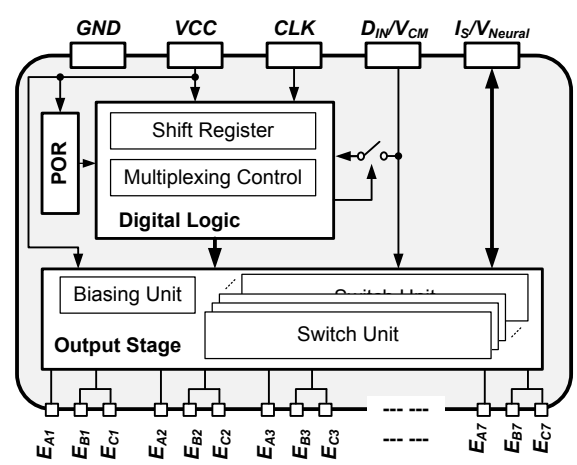

Figure 2. System diagram of the MNI ASIC.

stacked with a silicone layer in between. Each ASIC consists of seven tripolar electrode arrays, upon which seven microchannels are formed. The ASICs provide bidirectional multiplexing to each microchannel, and are powered and controlled by an implanted hybrid via a 5-line parallel bus. During stimulation, the hybrid provides a de stimulation current, $I_{S}$, to the MNI, where $I_{S}$ is converted into biphasic pulses by the switching activities in the MNI. During recording, the hybrid amplifies the received action potentials from the MNI by its built-in ENG preamplifier before forwarding the signals to the external controller. The hybrid connects to an external controller (not shown) via a percutaneous connector where commands are sent at a rate between $35 \mathrm{kbit} / \mathrm{s}$ and $1.1 \mathrm{Mbit} / \mathrm{s}$. Switching from stimulation to recording reroutes lines $I_{S} / V_{\text {Neural }}$ and $D_{I N} / V_{C M}$ to the inputs of the differential amplifier in the hybrid. Seven MNI ASICs are arranged in parallel with the 5-line parallel bus (GND, $\left.V C C, C L K, D_{I N} / V_{C M}, I_{S} / V_{\text {Neural }}\right)$. During recording a remote reference electrode and $I_{S} / V_{\text {Neural }}$ are biased to $V_{\text {Ref }}=1.65 \mathrm{~V}$ (midpoint of $V C C$ at $3.3 \mathrm{~V}$ ); the reference electrode acts to bias $D_{I N} / V_{C M}$.

\section{B. ASIC Design}

1) ASIC Architecture: The system diagram of the MNI ASIC is shown in Fig. 2. Each ASIC is connected to the 5-line parallel bus through five pads, where $D_{I N} / V_{C M}$ is used as both the data input, and the output for the common reference voltage, $V_{C M}$, for recording. Another shared pad, $I_{S} / V_{\text {Neural }}$, is used as current input during stimulation and neural signal output during recording. When powered up, an on-chip power-on-reset (POR) unit sets $D_{I N} / V_{C M}$ as the data input and disconnects all electrodes. Commands coming in from the data communication pads, $C L K$ and $D_{I N}$, are shifted into the digital logic where the multiplexing control activates the switch units in the output stage to connect the electrodes in the specified microchannels for either stimulation or recording.

2) Multiplexing Control: Each microchannel on the MNI ASIC comprises three electrode pads: $E_{A i}, E_{B i}$ and $E_{C i}$, arranged in a tripole, where $E_{A i}$ is located at the centre of the microchannel, and $E_{B i}$ and $E_{C i}$ on both sides at an equal pitch, as shown in Fig. 3. $E_{B i}$ and $E_{C i}$ are connected together to reduce inter-channel interference. The multiplexing operates in three modes: stimulation, recording and channel leak test. In the stimulation mode, one microchannel is activated at a time with the inner electrode $E_{A i}$ and the outer electrodes $E_{B i} / E_{C i}$ switching alternately between the input current $I_{S}$ and $G N D$, to create

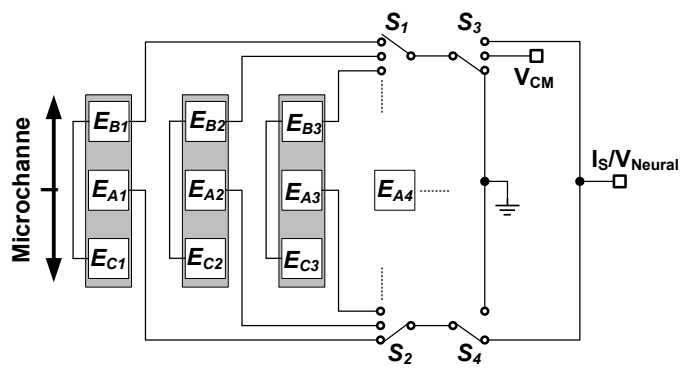

Figure 3. Illustration of the electrode multiplexing.

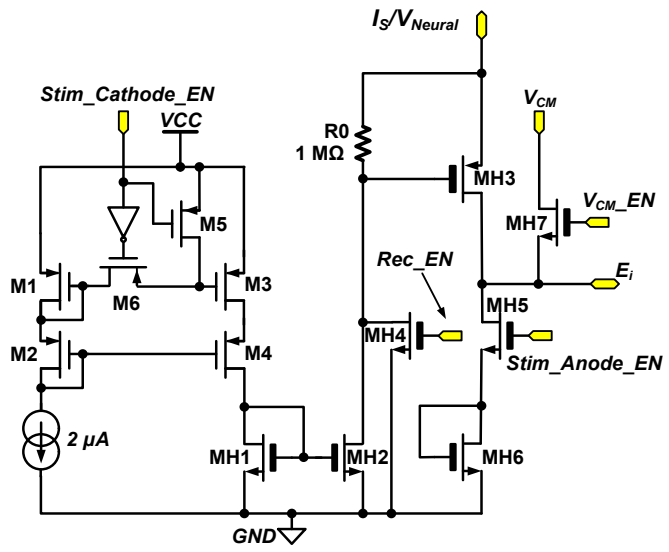

Figure 4. Schematic of the switch unit.

biphasic stimulus pulses. In the recording mode, the activation is also one microchannel at a time with $E_{A i}$ connected to the output, $V_{\text {Neural }}$, and the outer electrodes to the common voltage output, $V_{C M}$, with the connection between the $D_{I N} / V_{C M}$ pad and the digital logic turned off. Both $V_{\text {Neural }}$ and $V_{C M}$ are biased to $1.65 \mathrm{~V}$ and lead to the amplifier inputs in the hybrid. To exit the recording mode, a POR is needed to restore the connection between $D_{I N} / V_{C M}$ and the logic. In the channel leak test mode, two adjacent channels are activated at a time, with $\left(E_{A i}, E_{B i}, E_{C i}\right)$ connected to $I_{S}$ and $\left(E_{A i+1}, E_{B i+1}, E_{C i+1}\right)$ to $G N D$. This mode is for testing current leakage between the microchannels after the MNI assembly. Multiplexing is commanded by data frames via the communication pads.

3) Output Stage: The output stage consists of a biasing unit and 14 identical switch units ( 7 for $E_{A i}, 7$ for $E_{B i}$ and $E_{C i}$ ) for flexible channel multiplexing. The schematic of the switch unit is shown in Fig. 4. Four control signals operate a switch network to connect the electrode pad, $E_{i}$, to either $I_{S}$ or $G N D$ in the stimulation and channel leak test modes, or to either $V_{\text {Neural }}$ or $V_{C M}$ in the recording mode. The switches are implemented with high voltage MOSFETs in AMS HV $0.35-\mu \mathrm{m}$ CMOS technology. In the stimulation mode, high compliance voltage $(\leq 40 \mathrm{~V})$ may be required at $I_{S}$ due to the high impedance in a microchannel. As a result, conventional complementary CMOS switches are not suitable because the maximum allowed gatesource voltage is only $18 \mathrm{~V}$ for these HV MOSFETs. To ensure the safety of the transistors, a current-biased level shifter is implemented [10]. As shown in Fig. 4, once $E_{i}$ to this switch unit is specified as a cathode for stimulation, the control signal Stim_Cathode_EN turns on transistor M3 allowing a current of 


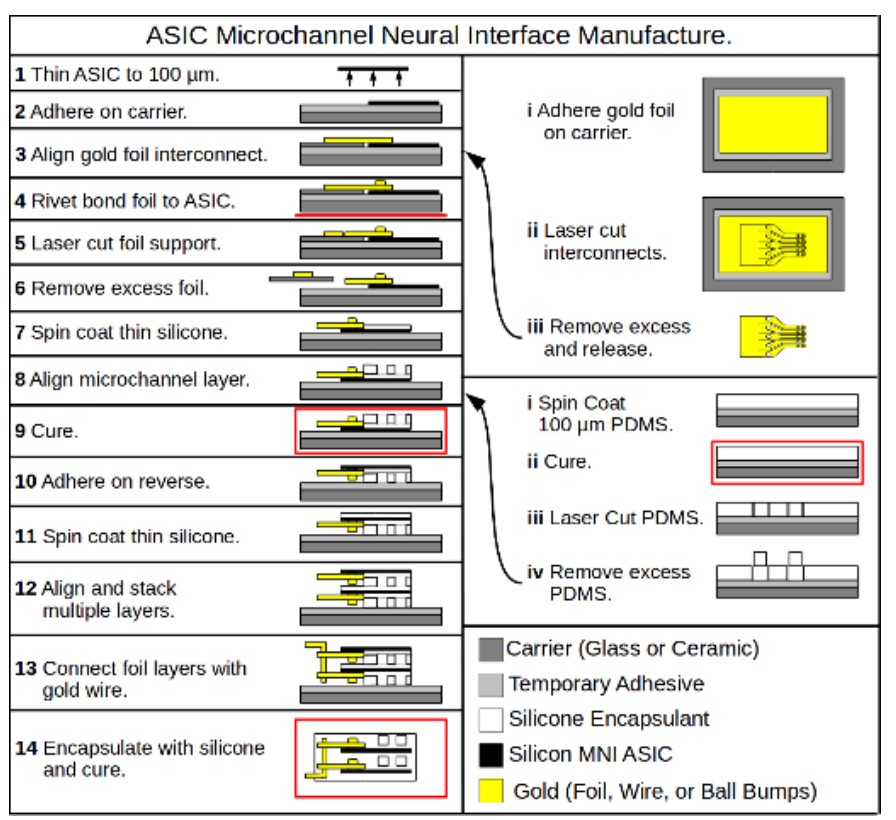

Figure 5. MNI manufacture from stacked MNI ASICs.

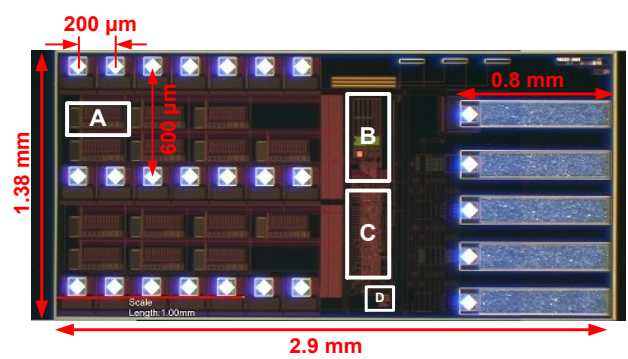

Figure 6. Die microphotograph of the MNI ASIC, including 14 identical switch unit (A), the biasing unit (B), the digital logic (C), the POR (D), five connecting pads and 21 electrode pads for 7 microchannels.

$2 \mu \mathrm{A}$ to pass through $\mathrm{MH} 2$, which generates a $2 \mathrm{~V}$ drop across R0, thus MH3 is safely turned on. If $E_{i}$ is specified as an anode, MH5 is turned on by Stim Anode EN to allow the stimulation current to flow to GND. In the recording mode, both $\mathrm{MH} 2$ and MH5 are turned off. For the switch unit to the inner electrodes, since the voltage at $V_{\text {Neural }}$ is biased at the midpoint of the $3.3 \mathrm{~V}$ supply, MH3 is directly turned on by connecting its gate to GND through MH4. For the outer electrodes, both MH3 and MH4 are turned off, and the electrodes are connected to $V_{C M}$ through MH7. The biasing unit generates a $2 \mu \mathrm{A}$ bias current for each of the switch units.

\section{Stacked Microchannel ASIC Assembly}

The complete MNI is assembled following a method used previously for passive conductors [8], [11]. The details of the procedure are summarized in Fig. 5. In general, the ASICs were first thinned to $100 \mu \mathrm{m}$ and attached to a carrier where the electrode pads was electroplated with gold, and laser-cut gold foil ribbons were rivet bonded to the five connection pads. The ASICs were then silicone encapsulated by spin coating with the electrode pads exposed. A silicone microchannel layer was formed by laser-cutting $100 \mu \mathrm{m}$ PDMS on a carrier. After removing excess PDMS, the microchannel layer was aligned

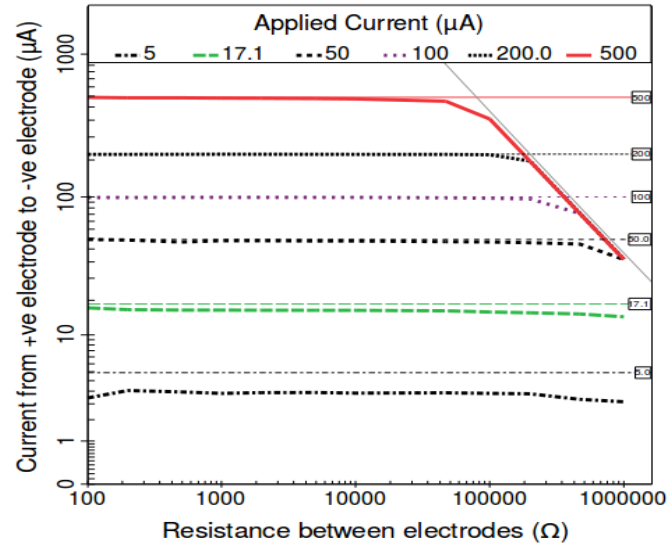

Figure 7. Current controlled stimulation. The observed current $(\mu \mathrm{A})$ between the cathode and anode during stimulation with a range of resistances between the electrodes (discounting saturation due to the $40 \mathrm{~V}$ voltage limit imposed).

with

$\mathrm{MNI}$
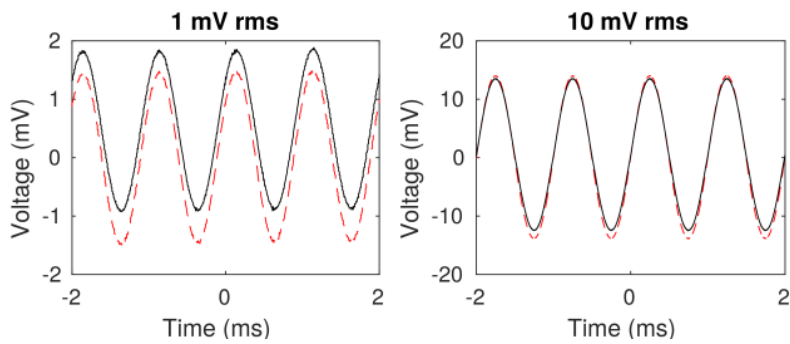

Figure $8.1 \mathrm{kHz}$ sine wave signals applied at $E_{A i}$ (red dashed line) and recorded at $I_{s} / V_{\text {Neural }}$ (black line).

to the ASIC and bonded. After curing, the bottom side of the MNI ASIC was also coated with thin silicone. By repeating this process, multiple layers of microchannels can be formed, as shown in steps $1-12$ in Fig. 5. Once the desired number of layers were achieved, vertical interconnections were formed between the gold foil ribbons using wire to form a vertical parallel bus, and then the MNI was silicone encapsulated to insulate the 5 connection lines, as shown in steps $13-14$. The red rectangle in Fig. 5 indicates a curing temperature of $150{ }^{\circ} \mathrm{C}$.

\section{MEASUREMENTS}

The prototype ASIC is implemented in the AMS $0.35 \mu \mathrm{m}$ high-voltage CMOS technology (H35B4D3). Fig. 6 shows the microphotograph of the ASIC. The total size of the die is $2.9 \mathrm{~mm} \times 1.38 \mathrm{~mm}$, including a length of $0.8 \mathrm{~mm}$ for the five extended connection pads. Seven microchannels are fabricated on the ASIC with three electrode pads per channel in a pitch of $600 \mu \mathrm{m}$. The size of each electrode pad is $94 \mu \mathrm{m} \times 94 \mu \mathrm{m}$. The pitch between adjacent microchannels is $200 \mu \mathrm{m}$. A comparison with other chip designs for active electrodes is shown in Table I

\section{A. Power Consumption}

The power consumption of a single MNI ASIC in POR mode is $143 \mu \mathrm{W}(43.3 \mu \mathrm{A}$ with a $3.3 \mathrm{~V}$ supply), where all electrodes are connected to GND. When switched to stimulate on one channel (cathodically or anodically), power consumption is 149 $\mu \mathrm{W}$. When all channels are connected for stimulation power 
TABLE I COMPARISON WITH OTHER WORK

\begin{tabular}{|c|c|c|c|c|c|c|c|c|}
\hline Reference & $\begin{array}{c}\text { CMOS } \\
\text { Process }\end{array}$ & $\begin{array}{c}\text { No. of } \\
\text { electrodes }\end{array}$ & $\begin{array}{c}\text { Die area } \\
\left(\mathrm{mm}^{2}\right)\end{array}$ & $\begin{array}{c}\text { Electrode } \\
\text { density }\end{array}$ & $\begin{array}{c}\text { No. of } \\
\text { wires }\end{array}$ & $\begin{array}{c}\text { Compliance } \\
\text { voltage }(\mathrm{V})\end{array}$ & $\begin{array}{c}\text { Channel } \\
\text { multiplexing }\end{array}$ & $\begin{array}{c}\text { Power } \\
\text { consumption }\end{array}$ \\
\hline [6] & $0.18 \mu \mathrm{m}$ & 5 & 1.753 & $2.85 / \mathrm{mm}^{2}$ & 3 & up to 25 & unidirectional & $114 \mu \mathrm{W}$ \\
\hline [14] & $1.5 \mu \mathrm{m}$ & 8 & 4.84 & $1.65 / \mathrm{mm}^{2}$ & $\mathrm{~N} / \mathrm{A}$ & 13 & unidirectional & $500 \mu \mathrm{W}$ \\
\hline [15] & $1.5 \mu \mathrm{m}$ & 64 & 21.16 & $3.02 / \mathrm{mm}^{2}$ & 2 & 5 & unidirectional & $8.25 \mathrm{~mW}$ \\
\hline [16] & $0.6 \mu \mathrm{m}$ & 12 & 27.3 & $0.44 / \mathrm{mm}^{2}$ & 5 & 18 & unidirectional & $30.11 \mathrm{~mW}$ \\
\hline This work & $0.35 \mu \mathrm{m}$ & 21 & 4 & $5.25 / \mathrm{mm}^{2}$ & 5 & up to 40 & bidirectional & $149.49 \mu \mathrm{W}$ \\
\hline
\end{tabular}

consumption is $237 \mu \mathrm{W}$, however this mode is not intended for use in practice. The recording and impedance test modes both consume $143 \mu \mathrm{W}$; thus power consumption for 7 stacked ASICs for a 49 channel MNI should be $1 \mathrm{~mW}$ to $1.1 \mathrm{~mW}$, dependent upon the mode.

\section{B. Stimulation}

The setup for nerve stimulation was tested. Current response under direct currents is shown in Fig. 7. The supply voltage was set to $40 \mathrm{~V}$ with six different levels of stimulation current at $I_{S} / V_{\text {Neural }}$. Variable resistors were used as dummy impedance between electrodes in a microchannel. It is shown in Fig. 7 that $1.44 \pm 0.55 \mu \mathrm{A}$ of the current supplied at $I_{S} / V_{\text {Neural }}$ is used to switch on the stimulating circuit and is therefore lost at the electrodes.

\section{Recording}

The electrodes were biased to $V_{\text {Ref }}(1.65 \mathrm{~V}$, i.e. $\mathrm{VCC} / 2)$ using an external bias source. The outer guard electrodes $\left(E_{B i} / E_{C i}\right)$ were biased by a $10 \mathrm{k} \Omega$ bias resistor representing a remote reference electrode, and a $47 \mathrm{k} \Omega$ impedance from $E_{A i}$ to $E_{B i} / E_{C i}$ was assumed. $I_{S} / V_{\text {Neural }}$ was also biased to $V_{\text {Ref. }} 1 \mathrm{kHz}$ sinusoids signals were applied at the electrodes and the outputs at $I_{S} / V_{\text {Neural }}$ and $D_{I N} / V_{C M}$ were recorded, as shown in Fig. 8.

\section{DISCUSSION AND CONCLUSION}

An ASIC design suitable for use in MNIs has been presented for stimulation and recording of peripheral nerve action potentials. Currents in the range required for stimulation in microchannels can be achieved with the MNI ASIC. Here, transfer of $1 \mathrm{mVp}$-p signals through the MNI ASIC has been shown. For use in reversible kilohertz frequency nerve conduction blocking when treating pain and incontinence, biphasic stimulus pulses of $1 \mathrm{kHz}$ to $100 \mathrm{kHz}$ are required [12]. With the current control system a maximum stimulus frequency of $50 \mathrm{kHz}$ is achievable; to achieve a $100 \mathrm{kHz}$ stimulus pulse a data rate in excess of $2 \mathrm{Mbit} / \mathrm{s}$ is required.

This ASIC makes possible an MNI comprising 7 layers and a total of 49 addressable microchannels, enabling recording and stimulation from 49 possible axon bundles. This represents an important increase in instrumented channel number over previous designs while reducing the number of connecting wires. Previous designs have electrodes present in up to 20 microchannels [6]. The assembled MNI will be tested on a regenerated nerve to demonstrate that it can both stimulate and record neural activity. In addition, such implant tests will help understand the materials aspects of using integrated circuits in intimate contact with body fluid and what device lifetime may be possible in the absence of a hermetic package [13].

\section{ACKNOWLEDGMENT}

Henry Lancashire thanks T. Perkins for helpful discussions and G. Blunn for support.

\section{REFERENCES}

[1] E. Biddiss, D. Beaton, and T. Chau, "Consumer design priorities for upper limb prosthetics," Disabil Rehabil Assist Technol, vol. 2, no. 6, pp. 346357, Jan. 2007.

[2] I. Dudkiewicz, R. Gabrielov, I. Seiv-Ner, G. Zelig, and M. Heim, "Evaluation of prosthetic usage in upper limb amputees," Disabil Rehabil, vol. 26, no. 1, pp. 60-63, Jan. 2004.

[3] G. S. Dhillon and K. W. Horch, "Direct neural sensory feedback and control of a prosthetic arm," IEEE Trans. Neural Systems Rehabil. Eng., vol. 13, no. 4, pp. 468-472, 2005.

[4] M. Ortiz-Catalan, R. Brånemark, B. Håkansson, and J. Delbeke, “On the viability of implantable electrodes for the natural control of artificial limbs: Review and discussion," Biomed Eng Online, vol. 11, p. 33, Jun. 2012.

[5] X. Navarro, T. Krueger, N. Lago, S. Micera, T. Stieglitz, and P. Dario, “A critical review of interfaces with the peripheral nervous system for the control of neuroprostheses and hybrid bionic systems", J. Peripheral Nervous System, vol. 10, no. 3, pp. 229-258, 2005.

[6] J. J. FitzGerald et al., "A regenerative microchannel neural interface for recording from and stimulating peripheral axons in vivo," J. Neural Eng., vol. 9, no. 1, p. 016010, Feb. 2012.

[7] J. J. Fitzgerald, S. P. Lacour, S. B. McMahon, and J. W. Fawcett, "Microchannels as axonal amplifiers," IEEE Trans. Biomed. Eng., vol. 55, no. 3, pp. 1136-1146, Mar. 2008.

[8] H. T. Lancashire et al., "Microchannel neural interface manufacture by stacking silicone and metal foil laminae," J. Neural Eng., vol. 13, no. 3, p. 034001, 2016.

[9] A. Srinivasan et al., "A regenerative microchannel device for recording multiple single-unit action potentials in awake, ambulatory animals," Eur J. Neurosci., vol. 43, no. 3, pp. 474-485, Feb. 2016.

[10] V. Giagka, et al., "An implantable versatile electrode-driving ASIC for chronic epidural stimulation in rats," IEEE Trans. Biomed. Circuits Syst., vol. 9, no. 3, pp. 387-400, Jun. 2015.

[11] H. T. Lancashire and N. Donaldson, "Vertical interconnects for stacked ASICs in soft encapsulant," MicroTech 2017, Didcot, UK, 2017.

[12] K. L. Kilgore, and N. Bhadra, "Reversible nerve conduction block using kilohertz frequency alternating current," Neuromodulation, vol. 17, no. 3, pp. 242-254-255, Apr. 2014.

[13] A. Vanhoestenberghe and N. Donaldson, "Corrosion of silicon integrated circuits and lifetime predictions in implantable electronic devices," $J$. Neural Eng., vol. 10, no. 3, p. 031002, 2013

[14] M. Sivaprakasam, et al., "A variable range bi-phasic current stimulus driver circuitry for an implantable retinal prosthetic device," IEEE J. Solid-State Circuits, vol. 40, no. 3, pp. 763-771, Mar. 2005.

[15] M. Ghovanloo and K. Najafi, "A wireless implantable multichannel microstimulating system-on-a-chip with modular architecture," IEEE Trans. Neural Syst. Rehabil. Eng., vol. 15, no. 3, pp. 449-457, Sep. 2007.

[16] X. Liu, et al., "Active Books: The design of an implantable stimulator that minimizes cable count using integrated circuits very close to electrodes," IEEE Trans. Biomed. Circuits Syst., vol. 6, no. 3, pp. 216-227, Jun. 2012. 